\title{
Disarming a Paradox of Validity
}

\author{
Hartry Field
}

\begin{abstract}
Any theory of truth must find a way around Curry's paradox, and there are well-known ways to do so. This paper concerns an apparently analogous paradox, about validity rather than truth, which JC Beall and Julien Murzi ("Two flavors of Curry's paradox") call the v-Curry. They argue that there are reasons to want a common solution to it and the standard Curry paradox, and that this rules out the solutions to the latter offered by most "naive truth theorists." To this end they recommend a radical solution to both paradoxes, involving a substructural logic, in particular, one without structural contraction.

In this paper I argue that substructuralism is unnecessary. Diagnosing the "v-Curry" is complicated because of a multiplicity of readings of the principles it relies on. But these principles are not analogous to the principles of naive truth, and taken together, there is no reading of them that should have much appeal to anyone who has absorbed the morals of both the ordinary Curry paradox and the second incompleteness theorem.
\end{abstract}

\section{Introduction}

Any theory of truth must deal in some way with Curry's paradox: what JC Beall and Julien Murzi [2] designate the $c$-Curry, where the prefix c- indicates that the paradox involves a conditional connective. The paradox is commonly dealt with either by restriction on some of the usual assumptions about truth, by restriction on some of the usual rules or metarules of classical logic, or by both. Beall and Murzi argue that there is an analogous paradox that they call the $v$-Curry, which does not involve a conditional at all but rather involves a validity predicate. They argue that the most popular solutions to the c-Curry do not extend to the v-Curry. They argue that we need a common solution and propose a radical one, involving a logic without structural contraction: roughly speaking, a logic where a conclusion $B$ can follow from an assumption $A$ taken twice without it following from $A$ taken once.

Received December 6, 2013; accepted March 20, 2014

First published online November 17, 2016

2010 Mathematics Subject Classification: Primary 03A99; Secondary 03B99, 03B47, 03F45

Keywords: Curry paradox, substructural logic, validity

(C) 2017 by University of Notre Dame 10.1215/00294527-3699865 


\title{
Disarming a Paradox of Validity
}

\author{
Hartry Field
}

\begin{abstract}
Any theory of truth must find a way around Curry's paradox, and there are well-known ways to do so. This paper concerns an apparently analogous paradox, about validity rather than truth, which JC Beall and Julien Murzi ("Two flavors of Curry's paradox") call the v-Curry. They argue that there are reasons to want a common solution to it and the standard Curry paradox, and that this rules out the solutions to the latter offered by most "naive truth theorists." To this end they recommend a radical solution to both paradoxes, involving a substructural logic, in particular, one without structural contraction.

In this paper I argue that substructuralism is unnecessary. Diagnosing the "v-Curry" is complicated because of a multiplicity of readings of the principles it relies on. But these principles are not analogous to the principles of naive truth, and taken together, there is no reading of them that should have much appeal to anyone who has absorbed the morals of both the ordinary Curry paradox and the second incompleteness theorem.
\end{abstract}

\section{Introduction}

Any theory of truth must deal in some way with Curry's paradox: what JC Beall and Julien Murzi [2] designate the $c$-Curry, where the prefix c- indicates that the paradox involves a conditional connective. The paradox is commonly dealt with either by restriction on some of the usual assumptions about truth, by restriction on some of the usual rules or metarules of classical logic, or by both. Beall and Murzi argue that there is an analogous paradox that they call the $v$-Curry, which does not involve a conditional at all but rather involves a validity predicate. They argue that the most popular solutions to the c-Curry do not extend to the v-Curry. They argue that we need a common solution and propose a radical one, involving a logic without structural contraction: roughly speaking, a logic where a conclusion $B$ can follow from an assumption $A$ taken twice without it following from $A$ taken once.

Received December 6, 2013; accepted March 20, 2014

First published online November 17, 2016

2010 Mathematics Subject Classification: Primary 03A99; Secondary 03B99, 03B47, 03F45

Keywords: Curry paradox, substructural logic, validity

(C) 2017 by University of Notre Dame 10.1215/00294527-3699865 


\title{
Disarming a Paradox of Validity
}

\author{
Hartry Field
}

\begin{abstract}
Any theory of truth must find a way around Curry's paradox, and there are well-known ways to do so. This paper concerns an apparently analogous paradox, about validity rather than truth, which JC Beall and Julien Murzi ("Two flavors of Curry's paradox") call the v-Curry. They argue that there are reasons to want a common solution to it and the standard Curry paradox, and that this rules out the solutions to the latter offered by most "naive truth theorists." To this end they recommend a radical solution to both paradoxes, involving a substructural logic, in particular, one without structural contraction.

In this paper I argue that substructuralism is unnecessary. Diagnosing the "v-Curry" is complicated because of a multiplicity of readings of the principles it relies on. But these principles are not analogous to the principles of naive truth, and taken together, there is no reading of them that should have much appeal to anyone who has absorbed the morals of both the ordinary Curry paradox and the second incompleteness theorem.
\end{abstract}

\section{Introduction}

Any theory of truth must deal in some way with Curry's paradox: what JC Beall and Julien Murzi [2] designate the $c$-Curry, where the prefix c- indicates that the paradox involves a conditional connective. The paradox is commonly dealt with either by restriction on some of the usual assumptions about truth, by restriction on some of the usual rules or metarules of classical logic, or by both. Beall and Murzi argue that there is an analogous paradox that they call the $v$-Curry, which does not involve a conditional at all but rather involves a validity predicate. They argue that the most popular solutions to the c-Curry do not extend to the v-Curry. They argue that we need a common solution and propose a radical one, involving a logic without structural contraction: roughly speaking, a logic where a conclusion $B$ can follow from an assumption $A$ taken twice without it following from $A$ taken once.

Received December 6, 2013; accepted March 20, 2014

First published online November 17, 2016

2010 Mathematics Subject Classification: Primary 03A99; Secondary 03B99, 03B47, 03F45

Keywords: Curry paradox, substructural logic, validity

(C) 2017 by University of Notre Dame 10.1215/00294527-3699865 


\title{
Disarming a Paradox of Validity
}

\author{
Hartry Field
}

\begin{abstract}
Any theory of truth must find a way around Curry's paradox, and there are well-known ways to do so. This paper concerns an apparently analogous paradox, about validity rather than truth, which JC Beall and Julien Murzi ("Two flavors of Curry's paradox") call the v-Curry. They argue that there are reasons to want a common solution to it and the standard Curry paradox, and that this rules out the solutions to the latter offered by most "naive truth theorists." To this end they recommend a radical solution to both paradoxes, involving a substructural logic, in particular, one without structural contraction.

In this paper I argue that substructuralism is unnecessary. Diagnosing the "v-Curry" is complicated because of a multiplicity of readings of the principles it relies on. But these principles are not analogous to the principles of naive truth, and taken together, there is no reading of them that should have much appeal to anyone who has absorbed the morals of both the ordinary Curry paradox and the second incompleteness theorem.
\end{abstract}

\section{Introduction}

Any theory of truth must deal in some way with Curry's paradox: what JC Beall and Julien Murzi [2] designate the $c$-Curry, where the prefix c- indicates that the paradox involves a conditional connective. The paradox is commonly dealt with either by restriction on some of the usual assumptions about truth, by restriction on some of the usual rules or metarules of classical logic, or by both. Beall and Murzi argue that there is an analogous paradox that they call the $v$-Curry, which does not involve a conditional at all but rather involves a validity predicate. They argue that the most popular solutions to the c-Curry do not extend to the v-Curry. They argue that we need a common solution and propose a radical one, involving a logic without structural contraction: roughly speaking, a logic where a conclusion $B$ can follow from an assumption $A$ taken twice without it following from $A$ taken once.

Received December 6, 2013; accepted March 20, 2014

First published online November 17, 2016

2010 Mathematics Subject Classification: Primary 03A99; Secondary 03B99, 03B47, 03F45

Keywords: Curry paradox, substructural logic, validity

(C) 2017 by University of Notre Dame 10.1215/00294527-3699865 


\title{
Disarming a Paradox of Validity
}

\author{
Hartry Field
}

\begin{abstract}
Any theory of truth must find a way around Curry's paradox, and there are well-known ways to do so. This paper concerns an apparently analogous paradox, about validity rather than truth, which JC Beall and Julien Murzi ("Two flavors of Curry's paradox") call the v-Curry. They argue that there are reasons to want a common solution to it and the standard Curry paradox, and that this rules out the solutions to the latter offered by most "naive truth theorists." To this end they recommend a radical solution to both paradoxes, involving a substructural logic, in particular, one without structural contraction.

In this paper I argue that substructuralism is unnecessary. Diagnosing the "v-Curry" is complicated because of a multiplicity of readings of the principles it relies on. But these principles are not analogous to the principles of naive truth, and taken together, there is no reading of them that should have much appeal to anyone who has absorbed the morals of both the ordinary Curry paradox and the second incompleteness theorem.
\end{abstract}

\section{Introduction}

Any theory of truth must deal in some way with Curry's paradox: what JC Beall and Julien Murzi [2] designate the $c$-Curry, where the prefix c- indicates that the paradox involves a conditional connective. The paradox is commonly dealt with either by restriction on some of the usual assumptions about truth, by restriction on some of the usual rules or metarules of classical logic, or by both. Beall and Murzi argue that there is an analogous paradox that they call the $v$-Curry, which does not involve a conditional at all but rather involves a validity predicate. They argue that the most popular solutions to the c-Curry do not extend to the v-Curry. They argue that we need a common solution and propose a radical one, involving a logic without structural contraction: roughly speaking, a logic where a conclusion $B$ can follow from an assumption $A$ taken twice without it following from $A$ taken once.

Received December 6, 2013; accepted March 20, 2014

First published online November 17, 2016

2010 Mathematics Subject Classification: Primary 03A99; Secondary 03B99, 03B47, 03F45

Keywords: Curry paradox, substructural logic, validity

(C) 2017 by University of Notre Dame 10.1215/00294527-3699865 


\title{
Disarming a Paradox of Validity
}

\author{
Hartry Field
}

\begin{abstract}
Any theory of truth must find a way around Curry's paradox, and there are well-known ways to do so. This paper concerns an apparently analogous paradox, about validity rather than truth, which JC Beall and Julien Murzi ("Two flavors of Curry's paradox") call the v-Curry. They argue that there are reasons to want a common solution to it and the standard Curry paradox, and that this rules out the solutions to the latter offered by most "naive truth theorists." To this end they recommend a radical solution to both paradoxes, involving a substructural logic, in particular, one without structural contraction.

In this paper I argue that substructuralism is unnecessary. Diagnosing the "v-Curry" is complicated because of a multiplicity of readings of the principles it relies on. But these principles are not analogous to the principles of naive truth, and taken together, there is no reading of them that should have much appeal to anyone who has absorbed the morals of both the ordinary Curry paradox and the second incompleteness theorem.
\end{abstract}

\section{Introduction}

Any theory of truth must deal in some way with Curry's paradox: what JC Beall and Julien Murzi [2] designate the $c$-Curry, where the prefix c- indicates that the paradox involves a conditional connective. The paradox is commonly dealt with either by restriction on some of the usual assumptions about truth, by restriction on some of the usual rules or metarules of classical logic, or by both. Beall and Murzi argue that there is an analogous paradox that they call the $v$-Curry, which does not involve a conditional at all but rather involves a validity predicate. They argue that the most popular solutions to the c-Curry do not extend to the v-Curry. They argue that we need a common solution and propose a radical one, involving a logic without structural contraction: roughly speaking, a logic where a conclusion $B$ can follow from an assumption $A$ taken twice without it following from $A$ taken once.

Received December 6, 2013; accepted March 20, 2014

First published online November 17, 2016

2010 Mathematics Subject Classification: Primary 03A99; Secondary 03B99, 03B47, 03F45

Keywords: Curry paradox, substructural logic, validity

(C) 2017 by University of Notre Dame 10.1215/00294527-3699865 


\title{
Disarming a Paradox of Validity
}

\author{
Hartry Field
}

\begin{abstract}
Any theory of truth must find a way around Curry's paradox, and there are well-known ways to do so. This paper concerns an apparently analogous paradox, about validity rather than truth, which JC Beall and Julien Murzi ("Two flavors of Curry's paradox") call the v-Curry. They argue that there are reasons to want a common solution to it and the standard Curry paradox, and that this rules out the solutions to the latter offered by most "naive truth theorists." To this end they recommend a radical solution to both paradoxes, involving a substructural logic, in particular, one without structural contraction.

In this paper I argue that substructuralism is unnecessary. Diagnosing the "v-Curry" is complicated because of a multiplicity of readings of the principles it relies on. But these principles are not analogous to the principles of naive truth, and taken together, there is no reading of them that should have much appeal to anyone who has absorbed the morals of both the ordinary Curry paradox and the second incompleteness theorem.
\end{abstract}

\section{Introduction}

Any theory of truth must deal in some way with Curry's paradox: what JC Beall and Julien Murzi [2] designate the $c$-Curry, where the prefix c- indicates that the paradox involves a conditional connective. The paradox is commonly dealt with either by restriction on some of the usual assumptions about truth, by restriction on some of the usual rules or metarules of classical logic, or by both. Beall and Murzi argue that there is an analogous paradox that they call the $v$-Curry, which does not involve a conditional at all but rather involves a validity predicate. They argue that the most popular solutions to the c-Curry do not extend to the v-Curry. They argue that we need a common solution and propose a radical one, involving a logic without structural contraction: roughly speaking, a logic where a conclusion $B$ can follow from an assumption $A$ taken twice without it following from $A$ taken once.

Received December 6, 2013; accepted March 20, 2014

First published online November 17, 2016

2010 Mathematics Subject Classification: Primary 03A99; Secondary 03B99, 03B47, 03F45

Keywords: Curry paradox, substructural logic, validity

(C) 2017 by University of Notre Dame 10.1215/00294527-3699865 


\title{
Disarming a Paradox of Validity
}

\author{
Hartry Field
}

\begin{abstract}
Any theory of truth must find a way around Curry's paradox, and there are well-known ways to do so. This paper concerns an apparently analogous paradox, about validity rather than truth, which JC Beall and Julien Murzi ("Two flavors of Curry's paradox") call the v-Curry. They argue that there are reasons to want a common solution to it and the standard Curry paradox, and that this rules out the solutions to the latter offered by most "naive truth theorists." To this end they recommend a radical solution to both paradoxes, involving a substructural logic, in particular, one without structural contraction.

In this paper I argue that substructuralism is unnecessary. Diagnosing the "v-Curry" is complicated because of a multiplicity of readings of the principles it relies on. But these principles are not analogous to the principles of naive truth, and taken together, there is no reading of them that should have much appeal to anyone who has absorbed the morals of both the ordinary Curry paradox and the second incompleteness theorem.
\end{abstract}

\section{Introduction}

Any theory of truth must deal in some way with Curry's paradox: what JC Beall and Julien Murzi [2] designate the $c$-Curry, where the prefix c- indicates that the paradox involves a conditional connective. The paradox is commonly dealt with either by restriction on some of the usual assumptions about truth, by restriction on some of the usual rules or metarules of classical logic, or by both. Beall and Murzi argue that there is an analogous paradox that they call the $v$-Curry, which does not involve a conditional at all but rather involves a validity predicate. They argue that the most popular solutions to the c-Curry do not extend to the v-Curry. They argue that we need a common solution and propose a radical one, involving a logic without structural contraction: roughly speaking, a logic where a conclusion $B$ can follow from an assumption $A$ taken twice without it following from $A$ taken once.

Received December 6, 2013; accepted March 20, 2014

First published online November 17, 2016

2010 Mathematics Subject Classification: Primary 03A99; Secondary 03B99, 03B47, 03F45

Keywords: Curry paradox, substructural logic, validity

(C) 2017 by University of Notre Dame 10.1215/00294527-3699865 


\title{
Disarming a Paradox of Validity
}

\author{
Hartry Field
}

\begin{abstract}
Any theory of truth must find a way around Curry's paradox, and there are well-known ways to do so. This paper concerns an apparently analogous paradox, about validity rather than truth, which JC Beall and Julien Murzi ("Two flavors of Curry's paradox") call the v-Curry. They argue that there are reasons to want a common solution to it and the standard Curry paradox, and that this rules out the solutions to the latter offered by most "naive truth theorists." To this end they recommend a radical solution to both paradoxes, involving a substructural logic, in particular, one without structural contraction.

In this paper I argue that substructuralism is unnecessary. Diagnosing the "v-Curry" is complicated because of a multiplicity of readings of the principles it relies on. But these principles are not analogous to the principles of naive truth, and taken together, there is no reading of them that should have much appeal to anyone who has absorbed the morals of both the ordinary Curry paradox and the second incompleteness theorem.
\end{abstract}

\section{Introduction}

Any theory of truth must deal in some way with Curry's paradox: what JC Beall and Julien Murzi [2] designate the $c$-Curry, where the prefix c- indicates that the paradox involves a conditional connective. The paradox is commonly dealt with either by restriction on some of the usual assumptions about truth, by restriction on some of the usual rules or metarules of classical logic, or by both. Beall and Murzi argue that there is an analogous paradox that they call the $v$-Curry, which does not involve a conditional at all but rather involves a validity predicate. They argue that the most popular solutions to the c-Curry do not extend to the v-Curry. They argue that we need a common solution and propose a radical one, involving a logic without structural contraction: roughly speaking, a logic where a conclusion $B$ can follow from an assumption $A$ taken twice without it following from $A$ taken once.

Received December 6, 2013; accepted March 20, 2014

First published online November 17, 2016

2010 Mathematics Subject Classification: Primary 03A99; Secondary 03B99, 03B47, 03F45

Keywords: Curry paradox, substructural logic, validity

(C) 2017 by University of Notre Dame 10.1215/00294527-3699865 


\title{
Disarming a Paradox of Validity
}

\author{
Hartry Field
}

\begin{abstract}
Any theory of truth must find a way around Curry's paradox, and there are well-known ways to do so. This paper concerns an apparently analogous paradox, about validity rather than truth, which JC Beall and Julien Murzi ("Two flavors of Curry's paradox") call the v-Curry. They argue that there are reasons to want a common solution to it and the standard Curry paradox, and that this rules out the solutions to the latter offered by most "naive truth theorists." To this end they recommend a radical solution to both paradoxes, involving a substructural logic, in particular, one without structural contraction.

In this paper I argue that substructuralism is unnecessary. Diagnosing the "v-Curry" is complicated because of a multiplicity of readings of the principles it relies on. But these principles are not analogous to the principles of naive truth, and taken together, there is no reading of them that should have much appeal to anyone who has absorbed the morals of both the ordinary Curry paradox and the second incompleteness theorem.
\end{abstract}

\section{Introduction}

Any theory of truth must deal in some way with Curry's paradox: what JC Beall and Julien Murzi [2] designate the $c$-Curry, where the prefix c- indicates that the paradox involves a conditional connective. The paradox is commonly dealt with either by restriction on some of the usual assumptions about truth, by restriction on some of the usual rules or metarules of classical logic, or by both. Beall and Murzi argue that there is an analogous paradox that they call the $v$-Curry, which does not involve a conditional at all but rather involves a validity predicate. They argue that the most popular solutions to the c-Curry do not extend to the v-Curry. They argue that we need a common solution and propose a radical one, involving a logic without structural contraction: roughly speaking, a logic where a conclusion $B$ can follow from an assumption $A$ taken twice without it following from $A$ taken once.

Received December 6, 2013; accepted March 20, 2014

First published online November 17, 2016

2010 Mathematics Subject Classification: Primary 03A99; Secondary 03B99, 03B47, 03F45

Keywords: Curry paradox, substructural logic, validity

(C) 2017 by University of Notre Dame 10.1215/00294527-3699865 


\title{
Disarming a Paradox of Validity
}

\author{
Hartry Field
}

\begin{abstract}
Any theory of truth must find a way around Curry's paradox, and there are well-known ways to do so. This paper concerns an apparently analogous paradox, about validity rather than truth, which JC Beall and Julien Murzi ("Two flavors of Curry's paradox") call the v-Curry. They argue that there are reasons to want a common solution to it and the standard Curry paradox, and that this rules out the solutions to the latter offered by most "naive truth theorists." To this end they recommend a radical solution to both paradoxes, involving a substructural logic, in particular, one without structural contraction.

In this paper I argue that substructuralism is unnecessary. Diagnosing the "v-Curry" is complicated because of a multiplicity of readings of the principles it relies on. But these principles are not analogous to the principles of naive truth, and taken together, there is no reading of them that should have much appeal to anyone who has absorbed the morals of both the ordinary Curry paradox and the second incompleteness theorem.
\end{abstract}

\section{Introduction}

Any theory of truth must deal in some way with Curry's paradox: what JC Beall and Julien Murzi [2] designate the $c$-Curry, where the prefix c- indicates that the paradox involves a conditional connective. The paradox is commonly dealt with either by restriction on some of the usual assumptions about truth, by restriction on some of the usual rules or metarules of classical logic, or by both. Beall and Murzi argue that there is an analogous paradox that they call the $v$-Curry, which does not involve a conditional at all but rather involves a validity predicate. They argue that the most popular solutions to the c-Curry do not extend to the v-Curry. They argue that we need a common solution and propose a radical one, involving a logic without structural contraction: roughly speaking, a logic where a conclusion $B$ can follow from an assumption $A$ taken twice without it following from $A$ taken once.

Received December 6, 2013; accepted March 20, 2014

First published online November 17, 2016

2010 Mathematics Subject Classification: Primary 03A99; Secondary 03B99, 03B47, 03F45

Keywords: Curry paradox, substructural logic, validity

(C) 2017 by University of Notre Dame 10.1215/00294527-3699865 


\title{
Disarming a Paradox of Validity
}

\author{
Hartry Field
}

\begin{abstract}
Any theory of truth must find a way around Curry's paradox, and there are well-known ways to do so. This paper concerns an apparently analogous paradox, about validity rather than truth, which JC Beall and Julien Murzi ("Two flavors of Curry's paradox") call the v-Curry. They argue that there are reasons to want a common solution to it and the standard Curry paradox, and that this rules out the solutions to the latter offered by most "naive truth theorists." To this end they recommend a radical solution to both paradoxes, involving a substructural logic, in particular, one without structural contraction.

In this paper I argue that substructuralism is unnecessary. Diagnosing the "v-Curry" is complicated because of a multiplicity of readings of the principles it relies on. But these principles are not analogous to the principles of naive truth, and taken together, there is no reading of them that should have much appeal to anyone who has absorbed the morals of both the ordinary Curry paradox and the second incompleteness theorem.
\end{abstract}

\section{Introduction}

Any theory of truth must deal in some way with Curry's paradox: what JC Beall and Julien Murzi [2] designate the $c$-Curry, where the prefix c- indicates that the paradox involves a conditional connective. The paradox is commonly dealt with either by restriction on some of the usual assumptions about truth, by restriction on some of the usual rules or metarules of classical logic, or by both. Beall and Murzi argue that there is an analogous paradox that they call the $v$-Curry, which does not involve a conditional at all but rather involves a validity predicate. They argue that the most popular solutions to the c-Curry do not extend to the v-Curry. They argue that we need a common solution and propose a radical one, involving a logic without structural contraction: roughly speaking, a logic where a conclusion $B$ can follow from an assumption $A$ taken twice without it following from $A$ taken once.

Received December 6, 2013; accepted March 20, 2014

First published online November 17, 2016

2010 Mathematics Subject Classification: Primary 03A99; Secondary 03B99, 03B47, 03F45

Keywords: Curry paradox, substructural logic, validity

(C) 2017 by University of Notre Dame 10.1215/00294527-3699865 


\title{
Disarming a Paradox of Validity
}

\author{
Hartry Field
}

\begin{abstract}
Any theory of truth must find a way around Curry's paradox, and there are well-known ways to do so. This paper concerns an apparently analogous paradox, about validity rather than truth, which JC Beall and Julien Murzi ("Two flavors of Curry's paradox") call the v-Curry. They argue that there are reasons to want a common solution to it and the standard Curry paradox, and that this rules out the solutions to the latter offered by most "naive truth theorists." To this end they recommend a radical solution to both paradoxes, involving a substructural logic, in particular, one without structural contraction.

In this paper I argue that substructuralism is unnecessary. Diagnosing the "v-Curry" is complicated because of a multiplicity of readings of the principles it relies on. But these principles are not analogous to the principles of naive truth, and taken together, there is no reading of them that should have much appeal to anyone who has absorbed the morals of both the ordinary Curry paradox and the second incompleteness theorem.
\end{abstract}

\section{Introduction}

Any theory of truth must deal in some way with Curry's paradox: what JC Beall and Julien Murzi [2] designate the $c$-Curry, where the prefix c- indicates that the paradox involves a conditional connective. The paradox is commonly dealt with either by restriction on some of the usual assumptions about truth, by restriction on some of the usual rules or metarules of classical logic, or by both. Beall and Murzi argue that there is an analogous paradox that they call the $v$-Curry, which does not involve a conditional at all but rather involves a validity predicate. They argue that the most popular solutions to the c-Curry do not extend to the v-Curry. They argue that we need a common solution and propose a radical one, involving a logic without structural contraction: roughly speaking, a logic where a conclusion $B$ can follow from an assumption $A$ taken twice without it following from $A$ taken once.

Received December 6, 2013; accepted March 20, 2014

First published online November 17, 2016

2010 Mathematics Subject Classification: Primary 03A99; Secondary 03B99, 03B47, 03F45

Keywords: Curry paradox, substructural logic, validity

(C) 2017 by University of Notre Dame 10.1215/00294527-3699865 


\title{
Disarming a Paradox of Validity
}

\author{
Hartry Field
}

\begin{abstract}
Any theory of truth must find a way around Curry's paradox, and there are well-known ways to do so. This paper concerns an apparently analogous paradox, about validity rather than truth, which JC Beall and Julien Murzi ("Two flavors of Curry's paradox") call the v-Curry. They argue that there are reasons to want a common solution to it and the standard Curry paradox, and that this rules out the solutions to the latter offered by most "naive truth theorists." To this end they recommend a radical solution to both paradoxes, involving a substructural logic, in particular, one without structural contraction.

In this paper I argue that substructuralism is unnecessary. Diagnosing the "v-Curry" is complicated because of a multiplicity of readings of the principles it relies on. But these principles are not analogous to the principles of naive truth, and taken together, there is no reading of them that should have much appeal to anyone who has absorbed the morals of both the ordinary Curry paradox and the second incompleteness theorem.
\end{abstract}

\section{Introduction}

Any theory of truth must deal in some way with Curry's paradox: what JC Beall and Julien Murzi [2] designate the $c$-Curry, where the prefix c- indicates that the paradox involves a conditional connective. The paradox is commonly dealt with either by restriction on some of the usual assumptions about truth, by restriction on some of the usual rules or metarules of classical logic, or by both. Beall and Murzi argue that there is an analogous paradox that they call the $v$-Curry, which does not involve a conditional at all but rather involves a validity predicate. They argue that the most popular solutions to the c-Curry do not extend to the v-Curry. They argue that we need a common solution and propose a radical one, involving a logic without structural contraction: roughly speaking, a logic where a conclusion $B$ can follow from an assumption $A$ taken twice without it following from $A$ taken once.

Received December 6, 2013; accepted March 20, 2014

First published online November 17, 2016

2010 Mathematics Subject Classification: Primary 03A99; Secondary 03B99, 03B47, 03F45

Keywords: Curry paradox, substructural logic, validity

(C) 2017 by University of Notre Dame 10.1215/00294527-3699865 


\title{
Disarming a Paradox of Validity
}

\author{
Hartry Field
}

\begin{abstract}
Any theory of truth must find a way around Curry's paradox, and there are well-known ways to do so. This paper concerns an apparently analogous paradox, about validity rather than truth, which JC Beall and Julien Murzi ("Two flavors of Curry's paradox") call the v-Curry. They argue that there are reasons to want a common solution to it and the standard Curry paradox, and that this rules out the solutions to the latter offered by most "naive truth theorists." To this end they recommend a radical solution to both paradoxes, involving a substructural logic, in particular, one without structural contraction.

In this paper I argue that substructuralism is unnecessary. Diagnosing the "v-Curry" is complicated because of a multiplicity of readings of the principles it relies on. But these principles are not analogous to the principles of naive truth, and taken together, there is no reading of them that should have much appeal to anyone who has absorbed the morals of both the ordinary Curry paradox and the second incompleteness theorem.
\end{abstract}

\section{Introduction}

Any theory of truth must deal in some way with Curry's paradox: what JC Beall and Julien Murzi [2] designate the $c$-Curry, where the prefix c- indicates that the paradox involves a conditional connective. The paradox is commonly dealt with either by restriction on some of the usual assumptions about truth, by restriction on some of the usual rules or metarules of classical logic, or by both. Beall and Murzi argue that there is an analogous paradox that they call the $v$-Curry, which does not involve a conditional at all but rather involves a validity predicate. They argue that the most popular solutions to the c-Curry do not extend to the v-Curry. They argue that we need a common solution and propose a radical one, involving a logic without structural contraction: roughly speaking, a logic where a conclusion $B$ can follow from an assumption $A$ taken twice without it following from $A$ taken once.

Received December 6, 2013; accepted March 20, 2014

First published online November 17, 2016

2010 Mathematics Subject Classification: Primary 03A99; Secondary 03B99, 03B47, 03F45

Keywords: Curry paradox, substructural logic, validity

(C) 2017 by University of Notre Dame 10.1215/00294527-3699865 


\title{
Disarming a Paradox of Validity
}

\author{
Hartry Field
}

\begin{abstract}
Any theory of truth must find a way around Curry's paradox, and there are well-known ways to do so. This paper concerns an apparently analogous paradox, about validity rather than truth, which JC Beall and Julien Murzi ("Two flavors of Curry's paradox") call the v-Curry. They argue that there are reasons to want a common solution to it and the standard Curry paradox, and that this rules out the solutions to the latter offered by most "naive truth theorists." To this end they recommend a radical solution to both paradoxes, involving a substructural logic, in particular, one without structural contraction.

In this paper I argue that substructuralism is unnecessary. Diagnosing the "v-Curry" is complicated because of a multiplicity of readings of the principles it relies on. But these principles are not analogous to the principles of naive truth, and taken together, there is no reading of them that should have much appeal to anyone who has absorbed the morals of both the ordinary Curry paradox and the second incompleteness theorem.
\end{abstract}

\section{Introduction}

Any theory of truth must deal in some way with Curry's paradox: what JC Beall and Julien Murzi [2] designate the $c$-Curry, where the prefix c- indicates that the paradox involves a conditional connective. The paradox is commonly dealt with either by restriction on some of the usual assumptions about truth, by restriction on some of the usual rules or metarules of classical logic, or by both. Beall and Murzi argue that there is an analogous paradox that they call the $v$-Curry, which does not involve a conditional at all but rather involves a validity predicate. They argue that the most popular solutions to the c-Curry do not extend to the v-Curry. They argue that we need a common solution and propose a radical one, involving a logic without structural contraction: roughly speaking, a logic where a conclusion $B$ can follow from an assumption $A$ taken twice without it following from $A$ taken once.

Received December 6, 2013; accepted March 20, 2014

First published online November 17, 2016

2010 Mathematics Subject Classification: Primary 03A99; Secondary 03B99, 03B47, 03F45

Keywords: Curry paradox, substructural logic, validity

(C) 2017 by University of Notre Dame 10.1215/00294527-3699865 


\title{
Disarming a Paradox of Validity
}

\author{
Hartry Field
}

\begin{abstract}
Any theory of truth must find a way around Curry's paradox, and there are well-known ways to do so. This paper concerns an apparently analogous paradox, about validity rather than truth, which JC Beall and Julien Murzi ("Two flavors of Curry's paradox") call the v-Curry. They argue that there are reasons to want a common solution to it and the standard Curry paradox, and that this rules out the solutions to the latter offered by most "naive truth theorists." To this end they recommend a radical solution to both paradoxes, involving a substructural logic, in particular, one without structural contraction.

In this paper I argue that substructuralism is unnecessary. Diagnosing the "v-Curry" is complicated because of a multiplicity of readings of the principles it relies on. But these principles are not analogous to the principles of naive truth, and taken together, there is no reading of them that should have much appeal to anyone who has absorbed the morals of both the ordinary Curry paradox and the second incompleteness theorem.
\end{abstract}

\section{Introduction}

Any theory of truth must deal in some way with Curry's paradox: what JC Beall and Julien Murzi [2] designate the $c$-Curry, where the prefix c- indicates that the paradox involves a conditional connective. The paradox is commonly dealt with either by restriction on some of the usual assumptions about truth, by restriction on some of the usual rules or metarules of classical logic, or by both. Beall and Murzi argue that there is an analogous paradox that they call the $v$-Curry, which does not involve a conditional at all but rather involves a validity predicate. They argue that the most popular solutions to the c-Curry do not extend to the v-Curry. They argue that we need a common solution and propose a radical one, involving a logic without structural contraction: roughly speaking, a logic where a conclusion $B$ can follow from an assumption $A$ taken twice without it following from $A$ taken once.

Received December 6, 2013; accepted March 20, 2014

First published online November 17, 2016

2010 Mathematics Subject Classification: Primary 03A99; Secondary 03B99, 03B47, 03F45

Keywords: Curry paradox, substructural logic, validity

(C) 2017 by University of Notre Dame 10.1215/00294527-3699865 


\title{
Disarming a Paradox of Validity
}

\author{
Hartry Field
}

\begin{abstract}
Any theory of truth must find a way around Curry's paradox, and there are well-known ways to do so. This paper concerns an apparently analogous paradox, about validity rather than truth, which JC Beall and Julien Murzi ("Two flavors of Curry's paradox") call the v-Curry. They argue that there are reasons to want a common solution to it and the standard Curry paradox, and that this rules out the solutions to the latter offered by most "naive truth theorists." To this end they recommend a radical solution to both paradoxes, involving a substructural logic, in particular, one without structural contraction.

In this paper I argue that substructuralism is unnecessary. Diagnosing the "v-Curry" is complicated because of a multiplicity of readings of the principles it relies on. But these principles are not analogous to the principles of naive truth, and taken together, there is no reading of them that should have much appeal to anyone who has absorbed the morals of both the ordinary Curry paradox and the second incompleteness theorem.
\end{abstract}

\section{Introduction}

Any theory of truth must deal in some way with Curry's paradox: what JC Beall and Julien Murzi [2] designate the $c$-Curry, where the prefix c- indicates that the paradox involves a conditional connective. The paradox is commonly dealt with either by restriction on some of the usual assumptions about truth, by restriction on some of the usual rules or metarules of classical logic, or by both. Beall and Murzi argue that there is an analogous paradox that they call the $v$-Curry, which does not involve a conditional at all but rather involves a validity predicate. They argue that the most popular solutions to the c-Curry do not extend to the v-Curry. They argue that we need a common solution and propose a radical one, involving a logic without structural contraction: roughly speaking, a logic where a conclusion $B$ can follow from an assumption $A$ taken twice without it following from $A$ taken once.

Received December 6, 2013; accepted March 20, 2014

First published online November 17, 2016

2010 Mathematics Subject Classification: Primary 03A99; Secondary 03B99, 03B47, 03F45

Keywords: Curry paradox, substructural logic, validity

(C) 2017 by University of Notre Dame 10.1215/00294527-3699865 


\title{
Disarming a Paradox of Validity
}

\author{
Hartry Field
}

\begin{abstract}
Any theory of truth must find a way around Curry's paradox, and there are well-known ways to do so. This paper concerns an apparently analogous paradox, about validity rather than truth, which JC Beall and Julien Murzi ("Two flavors of Curry's paradox") call the v-Curry. They argue that there are reasons to want a common solution to it and the standard Curry paradox, and that this rules out the solutions to the latter offered by most "naive truth theorists." To this end they recommend a radical solution to both paradoxes, involving a substructural logic, in particular, one without structural contraction.

In this paper I argue that substructuralism is unnecessary. Diagnosing the "v-Curry" is complicated because of a multiplicity of readings of the principles it relies on. But these principles are not analogous to the principles of naive truth, and taken together, there is no reading of them that should have much appeal to anyone who has absorbed the morals of both the ordinary Curry paradox and the second incompleteness theorem.
\end{abstract}

\section{Introduction}

Any theory of truth must deal in some way with Curry's paradox: what JC Beall and Julien Murzi [2] designate the $c$-Curry, where the prefix c- indicates that the paradox involves a conditional connective. The paradox is commonly dealt with either by restriction on some of the usual assumptions about truth, by restriction on some of the usual rules or metarules of classical logic, or by both. Beall and Murzi argue that there is an analogous paradox that they call the $v$-Curry, which does not involve a conditional at all but rather involves a validity predicate. They argue that the most popular solutions to the c-Curry do not extend to the v-Curry. They argue that we need a common solution and propose a radical one, involving a logic without structural contraction: roughly speaking, a logic where a conclusion $B$ can follow from an assumption $A$ taken twice without it following from $A$ taken once.

Received December 6, 2013; accepted March 20, 2014

First published online November 17, 2016

2010 Mathematics Subject Classification: Primary 03A99; Secondary 03B99, 03B47, 03F45

Keywords: Curry paradox, substructural logic, validity

(C) 2017 by University of Notre Dame 10.1215/00294527-3699865 